\title{
A Simulation Model for Risk Management Support in IT Outsourcing
}

\author{
Tarcio R. Bezerra ${ }^{1}$, Seth Bullock ${ }^{2}$ and Antão Moura ${ }^{1}$ \\ ${ }^{1}$ Computing Systems Department, Federal University of Campina Grande, Campina Grande, Brazil \\ ${ }^{2}$ Department of Eletronic and Computer Science, University of Southampton, Southampton, United Kingdom \\ tarcio@copin.ufcg.edu.br,sgb@ecs.soton.ac.uk,antao@dsc.ufcg.edu.br
}

Keywords: $\quad$ IT Outsourcing, IT Capabilities, Risk Management, System Dynamics Simulation

\begin{abstract}
IT Outsourcing (ITO) is the practice to delegate organizational IT functions to a third party. However, this practice introduces important risks for customer organizations. We have developed a system dynamics simulation model to support ITO decision making that considers a dynamic and integrated view of capabilities management and benefits management. Two IT capabilities are modelled: Contract Monitoring (on the customer's side) and Service Delivery (on the supplier's side). In this paper the proposed model is used to assess the risks presented by a deficit in these capabilities. The results of our experiments indicate that the lack of contract monitoring capability in ITO contracting organizations directly impacts on service conclusion time and influences the cost of contract management, which is an important risk factor related to exceeding the ITO budget. It was also found that low levels of service delivery capability in the supplier most significantly impact the cost of rework and the number of penalties. These factors influence the profitability of the supplier and may induce it to abandon the contract prematurely.
\end{abstract}

\section{INTRODUCTION}

When an organization doesn't have the internal IT capabilities required for the provision of all of its IT services, it must look for external organizations able to fill the gap (Barney, 1991). This practice is named Information Technology Outsourcing - ITO.

Outsourcing is often used to transfer risk to third parties. However, this practice introduces new risks for customer organizations as well. The loss of internal technical skills is an important (new) risk factor for organizations embarking on an ITO initiative (Ngwenyama and Sullivan, 2007; Martens and Teutenberg, 2009). The goal of ITO is not to lose control of IT, given the importance and centrality that it typically has for the outsourcing business. This concern should be reflected in the various phases of the outsourcing cycle. However, there is no clarity on the part of managers on how to mitigate this risk in a rational and balanced way, without compromising the potential benefits of outsourcing (Lacity and Willcocks, 2009).

The specialized literature offers many conceptual articles that identify lists of ITO risks or develop
ITO risk models and empirical papers that address specific ITO risks, risk measurement (Bahli and Rivald 2005) and risk management strategies (Lacity and Willcocks, 2009).

On the other hand however, there are still gaps to be filled by tools and models that help managers to decide which capabilities to develop and / or maintain internal to their organizations, in which quantity or magnitude and how such capabilities behave in a dynamic scenario of constant interaction between internal IT and vendor's teams.

The model to support decision making and ITO capabilities management proposed in (Bezerra et al., 2014) captures the dynamics of interaction between the ITO contract monitoring capability of contracting organizations and the service delivery capability of supplier organizations. Here we expand on this ITO decision support model and apply it to assess risks in the ITO contract monitoring process, enabling the model to support risk-based decisions. We apply the risk assessment procedure proposed in (Pfahl, 2005) in the context of a Brazilian state tax and finance agency (SEFAZ-AL) to analyze the impact of two kinds of risk (lack of contract 
monitoring capability in ITO customers and lack of service delivery capability in suppliers) on the ITO budget, on the deadline for completion of services and on the relationship between contractor and supplier.

\section{RELATED WORK}

The term risk is used in a variety of settings, and can take on different meanings. In ISO / DIS 31000 (2008), risk is the effect of uncertainty on objectives, where an effect is a deviation from the expected outcome (positive and / or negative). In the scope of our work, we are interested in studying the risks of undesirable outcomes. Formally, the risk exposure is defined as RE $=[$ Prob (UO) $x$ Loss (UO)], where $\mathrm{RE}$ is the risk exposure, Prob (UO) is the probability of an undesirable outcome, and Loss (UO) is the loss due to an undesirable outcome.

Risk management consists of making decisions about management and investment based on evaluating the threats and vulnerabilities that apply to the area or the process of interest. A generic risk management process includes the following steps (ISO / DIS 31000, 2008): 1) Context establishment; 2) Risks identification; 3) Risk assessment (the process of measuring the level of risk, expressed in terms of the combination of consequences and likelihood); 4) Risk evaluation; 5) Risk treatment/control. Our work is focused on applying steps 1,2 and 3 to ITO scenarios.

Risk management in ITO is a topic that has been extensively studied for decades and is still a topic of great relevance. Within a recent review of ITO (Lacity and Willcocks, 2010), 36 of the 164 works cited specifically address ITO risk management. Among these articles, Earl (1996) identifies a list of risk factors, Osei-Bryson and Ngwenyama (2006) develop risk models, Ngwenyama and Sullivan (2006; 2007) incorporate strategies for risk mitigation contracts, Willcocks and Lacity (2000) develop empirical research and Bahli and Rivald (2005) focus on risk measurement.

Among the extensive list of risk factors identified in ITO by several authors, the lack of essential IT capabilities by customers and suppliers is almost ubiquitous (Martens and Teutenberg, 2009; Lacity and Willcocks, 2009-2010).

The literature on ITO shows the strong relationship between the contracting organizations capabilities and the expected outcomes of outsourcing initiatives (Lacity and Willcocks, 20092010): the capability to manage vendors, to negotiate contracts and technical / methodological capability in information systems development are strongly related to the ITO success. In his review of the literature on IT capabilities, Jorfi (2011) points to a positive relationship between IT capabilities and corporate strategic alignment.

Martens and Teuteberg (2009) review 97 articles focused on ITO risk management. They summarise the main ITO risk factors and the impacts generated by them, categorize these factors and use them to build more complex risk and impact factors. The authors also associate these factors with related stages of a typical ITO life cycle.

In general, there are two methods that can be used to measure risk: quantitative and qualitative. In the quantitative method, the risk metric is calculated using a methodology that attempts to quantify numerically the associated risk components. As a result, the risk could be represented in terms of potential financial loss, for instance. In the qualitative method, a subjective scale, such as low, medium and high, is used to estimate the components of risk. In this type of analysis, the results depend heavily on the knowledge of the experts that assign values to the risk components.

The use of purely quantitative approaches is extremely difficult and costly. Among the main challenges are the lack of information records and the difficulty in estimating costs. There is great difficulty for organizations in producing statistics because of the lack of accurate records. This difficulty affects two components of risk: estimates concerning the likelihood of an event and impact of an event.

Our work uses a quantitative method to calculate risk exposure, based on a quantitative system dynamics simulation model of the contract monitoring process, where impact factors are calculated from differential equations and probabilities can be calculated from the results of multivariate sensitivity analyses. We believe that a quantitative approach, despite the already outlined difficulties in adopting it, has a more objective power to communicate risks to the decision makers. This is of particular interest for business process managers wishing to make informed decisions based on quantitative (financial) values, especially in the case where risk treatment involves financial expenditures. To argue that an investment of some thousand dollars will avoid a loss of some million dollars is a clearer way to inform about risks than saying that a "very high" risk will be mitigated. Quantitative approaches can also encourage organizations to implement measurement programs 
to $\log$ quantitative empirical data. Furthermore, for comparison purposes - i.e., when one needs to compare (and select between) alternatives A and B, a quantitative approach provides a useful yardstick which may not need to be absolutely precise but just "relatively" precise (worse alternatives provide worse quantities). Even if errors are present in the estimated results (and they usually are), the quantitative approach is an efficient decision support tool for comparison and selection purposes.

Software simulation modeling has been extensively used in risk management applied to various sectors of knowledge, industry and services over the years. Some of these models use a static approach and others capture the dynamics of the processes to which they apply. Both approaches can stochastically generate values for risk factors as a strategy for representing uncertainty. Our adoption of system dynamics at the expense of other simulation techniques is that it is a holistic approach that is not limited to mono-causality relationships, but allows one to represent a complex network of inter-dependencies among risk and impact factors, including when impact factors are fed back to the system as risk factors.

The model proposed by us aims to be a tool for supporting decisions in ITO and in managing capabilities directly involved in the ITO process taking into account business benefits realization. In this direction, we have sought inspiration from system dynamics simulation models applied to project management in general and in particular to software engineering projects (Abdel-Hamid and Madnick, 1991; Lin et al., 1997; Garousi et al., 2009) and also applied to decision making in people management (Costa et al., 2013). One use case for the proposed model is taking risk-based decisions, considering the ITO risk factors and impacts that can be represented within the scope of the model. Risk assessment procedures to be applied to pre-existing system dynamics models are proposed in Houston et al. (2001) and Pfahl (2005) and influenced our work. There are very few examples of simulation-based ITO risk management research. Gui-sem and Xiangyang (2010) present a model structure for risk analysis. Our work differs in that our approach is quantitative, and we focus on the risks related to the IT capabilities involved in the ITO process.

Further down the ITO lifecycle come the stages of evaluation and treatment of risks. These involve decisions made by the model user supported by the outputs of the simulation model presented here combined with decision-making frameworks. As an example, Tan et al. (2010) proposes the use of a decision tree to evaluate the outputs of a system dynamics model applied to project risk management. These later stages however, are outside the scope of this paper - which concentrates on developing and applying a system dynamics simulation model to ITO risk assessment.

\section{A SIMULATION MODEL}

Measuring IT capabilities quantitatively in order to properly allocate resources to better achieve planned results (e.g. project objectives) is still a challenging problem, especially with regard to human resource skills and the impact of the tools and techniques used to support IT functions. However, there is a lack of tools and models that help managers to make decisions about capabilities management.

The loss of internal technical skills is an important risk factor for organizations embarking on an ITO initiative. However, there is little clarity for managers seeking to mitigate this risk in a rational and balanced way, without compromising the potential benefits that outsourcing can provide.

\subsection{Model Objectives}

We have developed a system dynamics simulation model to support ITO decision making that considers a dynamic and integrated view of capabilities management and benefits management.

Two IT capabilities are modeled here: Contract Monitoring - a core capability in the context of outsourcing which mediates all interactions between client and vendor capabilities; and, Service Delivery - a generic single point of contact for IT services.

The objective of this paper is to use the proposed model to assess the risks presented by deficits in these capabilities on the contractor and supplier sides, including the risk of a premature contract termination. Due to space limitations, this paper identifies risks only without discussing ways of mitigating them and details the model's implementation, which is somewhat complex, only to the extent of informing on its main modules and output.

\subsection{Architecture and Entities}

Our model has a large number of parameters, divided into four distinct categories: input, calibration, mediation and output.

Input parameters characterize the benefits and performance metrics to be achieved, the IT resources 
available within the organization and the IT demand characteristics. Calibration parameters are used to tune the model's behavior to match the scenarios being simulated. Mediation parameters represent intermediate information obtained from the entries, from calibration and, in situations involving feedback loops, from output parameters, e.g., IT capabilities and second-level performance targets (desired workforce, desired skill level). Output parameters are values arising from the dynamic cause-effect relationships between model input, calibration and mediator parameters. The model produces outputs that reflect the expected performance of IT resources (in terms of cost, quality, resource consumption) in response to submitted inputs.

\subsubsection{Model Views}

For clarity, maintainability and reusability, the model was segmented into "views", reflecting the organization of policies captured in the modeling phase (financial management; demand management; capability forecasting \& planning; sourcing management; insourced capabilities management; outsourced capabilities management; contract monitoring of IT processes/functions). The views that highlight the core concepts of our risk assessment are detailed below.

\subsubsection{Model Parameters}

The most important input (I), calibration (C) and output $(\mathrm{O})$ parameters are listed in Table 1 and will be detailed in section 5 .

Table 1: Main model parameters.

\begin{tabular}{|l|c|c|}
\hline Parameter & Unit & Type \\
\hline Task (SU = Service Units) & SU & I \\
\hline Task Conclusion Time & Days & I \\
\hline ITO Budget & $\$$ & I \\
\hline Initial Available CM WorkForce (WF) & Persons & I \\
\hline Initial Average CM Skill Level & - & I \\
\hline SD SLA & - & I \\
\hline Minimum SD Skill Level & - & I \\
\hline Time to Adjust CM WF & Days & C \\
\hline Time to Adjust CM Skill Level & Days & C \\
\hline CM Materials Effectiveness & - & C \\
\hline CM Intangible Effectiveness & - & C \\
\hline Time to adjust SD Productivity & Days & C \\
\hline $\begin{array}{l}\text { Cumulative Cost of Insourced CM } \\
\text { Capability }\end{array}$ & $\$$ & O \\
\hline $\begin{array}{l}\text { Cumulative Cost of Outsourced SD } \\
\text { Capability }\end{array}$ & $\$$ & O \\
\hline SD Demand Conclusion Time & Day & O \\
\hline Cost of Rework & $\$$ & O \\
\hline Penalties for Rework & $\$$ & O \\
\hline
\end{tabular}

\subsubsection{Sourcing Management}

In the sourcing management view, one can decide whether a particular IT capability will be fully executed by the internal team or completely or partially outsourced. We capture this behavior in our model by dividing the diagram representing a given IT capability into two parts: the internal part, which allocates internal resources to build the capability (located in the Insourced Capabilities Management view) and the external part, located in the Outsourced Capabilities Management view, where additional supplier capability is requested and allocated. In the present view, the user can set up the model to choose the desired sourcing mode for each capability.

\subsubsection{Insourced Capabilities Management}

This view (see Figure 1) contains the diagrams representing the ITO contracting organization's side of the IT capabilities, among them the Contract Monitoring (CM) capability.

We consider that a capability is effectively a

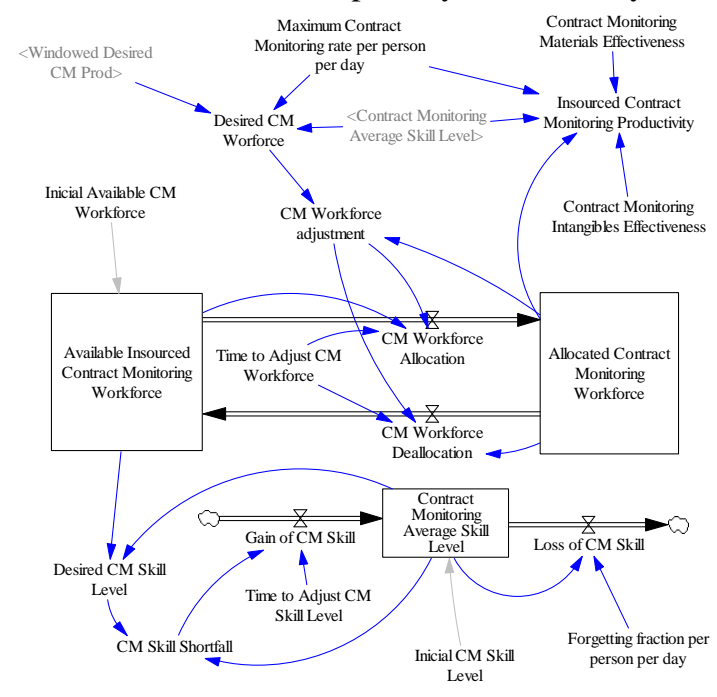

Figure 1: The Contract Monitoring Capability diagram.

productivity rate - i.e., the number of service units (SU) processed per day. Therefore, the Contract Monitoring capability is given by the variable Insourced CM Productivity, in SU/Day, which is calculated based on the productivity of the resources involved (people, material resources, intangible assets) using the following formula:

Insourced CM Productivity = Allocated Insourced CM Workforce x Maximum CM Rate per Person per Day x Average CM Skill Level x CM 


\section{Materials Effectiveness $\mathrm{x} \quad C M$ Intangibles Effectiveness}

Allocated Insourced CM Workforce represents the number of people allocated to monitor the contract; Maximum CM Rate per Person per Day is a constant used to represent the number of service units that an "optimally skilled" workforce is able to process in a day. The Average CM Skill Level parameter takes values between 0 and 1 and represents the average fraction of the optimal skill level presented by the internal staff. As our work is focused on human resources, the constant $C M$ Intangibles Effectiveness and CM Materials Effectiveness are just multipliers which represent the extent to which intangible and material resources empower staff productivity, respectively. The highlight of this view is the dynamic behavior of resources mobilized as capabilities governed by the need for productivity created by the service order to be processed (Windowed Desired CM Productivity) and subject to various operational delays (variables Time to Adjust CM Workforce, Time to Adjust CM Average Skill Level).

\subsubsection{Outsourced Capabilities Management}

The dynamic of the provider's capability behavior is captured in the diagram of Figure 2.

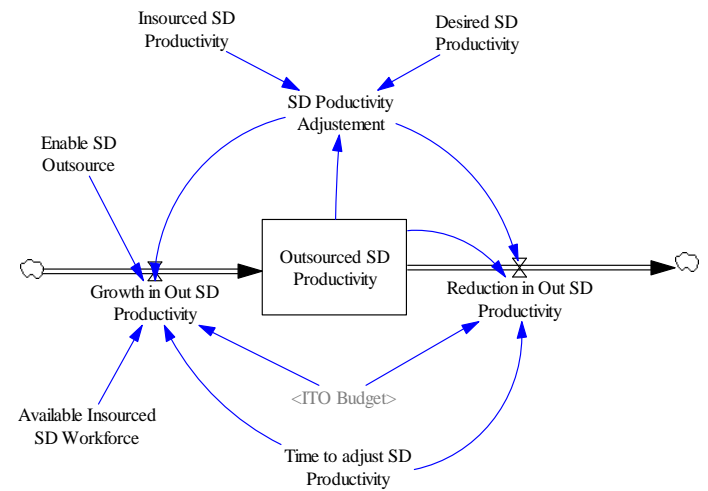

Figure 2: Supplier's Service Delivery Capability diagram.

If all of the organization's own resources have been allocated and even so the internal generated capability is insufficient to meet demand, if outsourcing is enabled and if there is available ITO budget, the provider's capability will be adjusted to the required levels subject to a required time for this adjustment. In our example, we use the Service Delivery (SD) generic capability.

\subsubsection{Contract Monitoring for Service Delivery}

This view (see Figure 3 ) captures the specifics of the demands flow between the customer's IT organization and the ITO provider. This flow reflects the contract monitoring process and the interaction between this capability and IT service delivery capability.

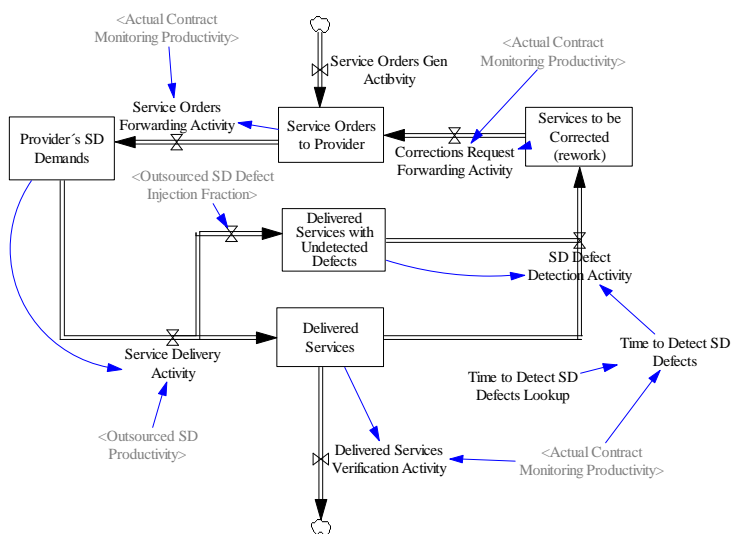

Figure 3: Interaction between Contract Monitoring and Outsourced Service Delivery capabilities.

The Actual Contract Monitoring Productivity variable moves the streams of new service orders and those on warranty (rework) from the customer's organization to the provider, as well as the flow of delivered services approval and defects detection.

The provider's capability to process the demands forwarded by the contractor is represented by the variable Outsourced SD Productivity. Outsourced SD Defect Injection Fraction represents the error generation rate in service delivery.

\section{ILLUSTRATION: ITO CONTRACT MONITORING AT SEFAZ-AL/BRAZIL}

Development and validation of the System Dynamics simulation model are ongoing in order to provide support to ITO decision making (Bezerra and Medeiros, 2013; Bezerra et al., 2014). The model has been in use at the Finance and Revenue Agency of Alagoas State, Brazil (SEFAZ-AL) which is for tax collection and financial control of the state administration. SEFAZ-AL has the largest IT department and the most important outsourcing activity in Alagoas, both in volume and in complexity (Cunha, 2011). Having gone through 
several generations of ITO, SEFAZ-AL has experienced various contract formats and models.

For the case study to be carried out in this section, we consider the scope of a contract that has been in operation for only a few months. Its purpose was to provide design and implementation services of new information systems (projects) and to maintain those already in production (continuous services). We had access to records of service orders (SO) performed during the contract. It is beyond the scope of this paper to analyze all of these service orders. We selected five SOs sent to the supplier since the inception of the contract in order to build the capacity plan for filling demands based on the contract parameters. To illustrate the use of our model for risk assessment, SO FIS07 was selected because it is the largest, with greatest potential for variation in the allocation of resources.

SO FIS07 has an expected workload of 5320 service units (SU), 163 days as expected conclusion time and its estimated cost is $\$ 267,560$. The anticipated contract management cost is $\$ 124,609$ and the cost of rework $\$ 3,240$. These two latter parameters were not originally registered by SEFAZ-AL, but were estimated using simulation.

In what follows we study how the proposed simulation model was applied to ITO risk management at SEFAZ-AL following a 5-step roadmap described in (Pfahl, 2005): 1) Defining the risk factors; 2) Defining impacts; 3) Defining the variation of risk factors; 4) Conducting sensitivity analysis; 5) Analyzing the results.

\subsection{Defining the Risk Factors}

A number of risk factors were selected based on important references in the ITO risk management literature (Earl, 1996; Rivard et al., 1998a-1998b; Martens and Teuteberg, 2009; Ngwenyama and Sullivan, 2006-2007; Bahli and Rivard, 2003). It is important to emphasize that the focus of the proposed model is on human resources management related to IT capabilities, the Contract Monitoring Capability in particular. Risk factors associated with attributes of the contract itself and of the relationship between contractor and supplier are outside the scope of the current model.

The causal relationships between risk factors and impact factors found in the cited references describe a complex network, which varies greatly in terms of granularity. In some cases, fine-grain risk factors are combined to form intermediate risk factors, which in turn relate to "final" impact factors or to impact factors which can themselves be considered as intermediate risk factors. For this reason, to relate the parameters of our model to the risk factors identified in the literature, we describe risk scenarios, as in (Bahli \& Rivard, 2003; Martens and Teuteberg, 2009), which can be interpreted as complex risk factors. For illustration purposes, the following two risk scenarios (and related model parameters) were selected:

RS1 - Insufficient capability of the contracting organization in monitoring ITO contracts. In this scenario, contracts based on performance metrics (quality, cost, reward, penalties, revenue, etc.) are highly impacted as it becomes costly and inefficient to measure such metrics, which seriously compromises the results of the ITO initiative.

In our work, we consider that the contract monitoring process involves the following skills: the capability to estimate effort levels and timelines for completing tasks, to have knowledge in IT outsourced function to check the delivered product or services, to operate the collection and recording of the contract performance indicators and to negotiate with the supplier in the event of dispute.

The less experience the customer has with the outsourced IT function, the more difficulty it will have in checking the delivered product or service and in estimating levels of effort and timelines for completing tasks. The less experience the customer has with contract management, the more difficulty it will have in negotiating with the supplier in the event of dispute, and in operationalizing the collection and recording of the performance indicators of the contract and with transferring the operation of the contracted service to the supplier's environment. Concerning other categories of resources, such as material and intangible ones, the lack of contract management tools may represent a bottleneck for managers' productivity, delay closure of invoices for payment, and cause difficulties in calculating penalties and in timely renegotiation and renewal of contracts. An incomplete or poorly detailed contract can generate dispute between contractor and supplier about scope and quality levels of the contracted service, methodology for calculating the quality and cost indicators, penalties and incentives. All these facts can lead, separately or in conjunction, to expected service conclusion time and cost misses; to acceptance of services with low quality level; and, to litigation with the supplier.

The contracts monitoring capability is represented in our model by a productivity rate $(\mathrm{CM}$ Productivity), measured in service units per day (SU 
/ Day) and calculated as a function of the parameters described below.

Associated model parameters are: Allocated CM Workforce (in Number of Persons): Human resources allocated to perform tasks related to the ITO contract monitoring; Initial Average CM Skill Level (no measurement units): Initial average skill level of internal staff allocated to the ITO contract monitoring in this function; Time to Adjust CM WF (Day): Operating delay in adjusting the contract monitoring human resources; Time to Adjust CM Skill Level (Day): Time required to absorb and apply training and/or to gain experience on contract monitoring; Time to Detect Defects (Day): Time required for a defect in a delivered service to be detected by the contract monitoring team. We modeled this parameter as a nonlinear function of the parameter CM Capability, so its behavior is endogenous; CM Materials Effectiveness (no measurement units): Effective contribution that material resources make to the contract monitoring capability; CM Intangible Effectiveness (no measurement units): Effective contribution that intangible resources make to the contract monitoring capability.

RS2 - Insufficient capability of the supplier to deliver the contracted service. Our work is focused on managing the contracting organization's resources and how to configure them to build IT capabilities. Therefore, we consider the supply-side capabilities in a consolidated basis (as a cloud). The supplier's service delivery capability involves the following skills: knowledge of the outsourced IT function and ability to deliver the product or service according to the performance parameters specified in the contract.

The less technical knowledge the supplier has on the outsourced IT function, the more he will fail to meet agreed performance requirements and this will directly affect the quality of the service delivered. Non compliant delivered services will be resubmitted to the vendor for corrections, which will delay the expected completion time for the service. The more re-work is generated, the more contract monitoring working hours will be consumed rechecking delivered services. This will increase contract monitoring costs. Rework over the parameters agreed in the contract will also generate penalties and extra operational costs for the supplier, decreasing its profitability and causing it to reduce interest in the contract.

The service delivery capability is represented in our model by a productivity rate (SD Productivity), measured in service units per day (SU / Day).
Associated model parameters are: Time to Adjust SD Productivity (Day): Operating delay to adjust the service delivery capability; Minimum SD Skill Level (no measurement units): Minimum average capability of the supplier's staff allocated to perform the outsourced IT function. This parameter influences the variable $S D$ Defect Injection Fraction; SD SLA (no measurement units): Service Level Agreement parameter is a real number between 0-1 that represents the minimum quality level of the delivered services. We say that a fraction (1-SD $S L A)$ of the delivered service units will have defects and will need to be reworked. This parameter doesn't influence penalties but influences the total cost of rework, which affects the supplier's profitability.

\subsection{Defining the Impacts}

The impact factors are attributes of the entities involved in IT services (client, provider, service itself), usually representing their performance indicators such as cost, completion time, quality level, satisfaction level, which are affected by changes in risk factors. Based on the same rationale given in section 5.1, here we describe impact scenarios as combinations of impact factors reaching certain conditions.

Based on the risk scenarios selected and on the causality relationships identified in the ITO risk management literature, the following impact scenarios are of interest.

IS1 - Exceed ITO budget. This impact scenario arises when the expected cost for service orders is exceeded. Associated model parameter is the Cumulative Cost of SD Capability (\$): Cost of the capability used to process all service units from a service order.

IS2 - Exceed the expected service conclusion time. This impact scenario when the expected conclusion time for service orders is exceeded. Associated model parameter is the: SD Demand Conclusion Time (Day): Number of days that a supplier requires to process a service order's service units completely.

IS3 - High contract management cost. The costs of internal resources are usually neglected or not computed in public sector outsourcing processes, where salaries of career employees are not considered as part of the projects/program budget (Carvalho, 2009). The effort (and cost) involved in managing contracts in Brazil typically represent between $30 \%$ and $40 \%$ of the related service cost 
(Carvalho, 2009). Exceeding this threshold means incurring additional management costs.

Associated model parameter is the Cumulative Cost of Insourced CM Capability (\$): Cost of the capability used for monitoring ITO contracts.

IS4 - Premature contract termination and service discontinuity or debasement. This impact scenario is more subjective. From the customer's point of view one can monitor indications that the supplier is losing money or is not achieving the profitability projected at the beginning of the contract. Therefore, the supplier has reduced interest in continuing the relationship. Thus, in a possible replacement scenario, services may be discontinued or have their quality compromised by the lack of resources for their proper functioning.

Associated model parameters are: Cost of Rework (\$) which is the cost to the supplier to correct detected defects in delivered services; and, Penalties for Rework (Number of Penalties) which is the number of penalties issued to the supplier upon reaching a contractually agreed rework index.

All "expected values" mentioned in the description of impact scenarios are established relative to a baseline. This baseline can be constructed from empirical data, interviews with experts or generated synthetically using simulation.

\subsection{Relationships between Risk and Impact Scenarios}

Figure 4 summarizes the cause-and-effect relations of risk and impact scenarios within the model.

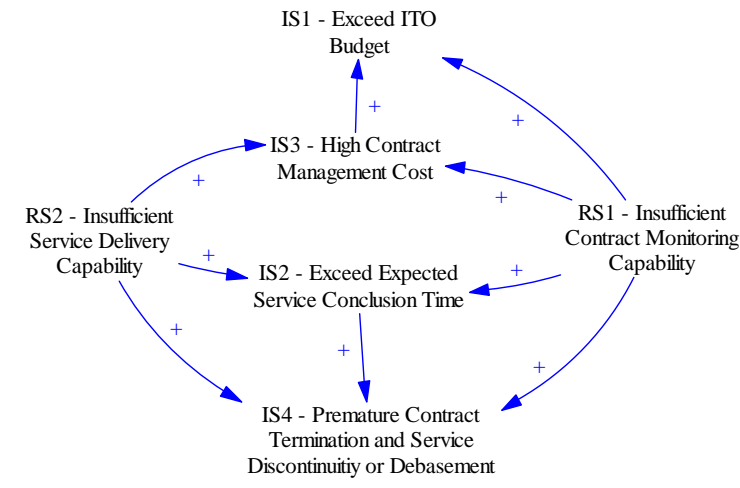

Figure 4: Cause-and-effect relationships between risk and impact scenarios.

These relations were established based on (Earl, 1996; Rivard et al, 1998a-1998b; Bahli and Rivard, 2003) and on interviews with experts from SEFAZ-
AL. Figure 4 includes hypotheses to be explored in the sensitivity analysis in 4.5 .

\subsection{Variation of Risk Factors}

The range of risk factor values reflects the uncertainty with which decision makers predict impacts. Such uncertainties are generated stochastically by varying the model input variables (risk factors) according to probability distribution functions. These functions are constructed based on empirical data and goodness-of-fit tests or the triangular probability distribution function is used with parameters estimated by experts. Here, we use estimates from SEFAZ-AL experts. It is important to emphasize that the subjective estimation of numerical parameters made by experts based on their experience and knowledge does not violate the quantitative nature of our approach. Also note that history (information in logs), conditions (such as physical, temporal or financial limitations) and guidelines (such as those established in corporate policies) may reduce the "subjectivity" in providing estimates.

To better understand the impacts caused by variation in risk factors, these variations will be divided into (a) Contractor conditions and; (b) Supplier conditions, as laid out in Table 2.

Table 2: Risk factor variation.

\begin{tabular}{|l|c|c|c|}
\hline \multirow{2}{*}{ Parameters } & \multicolumn{3}{c|}{ Estimated values } \\
\cline { 2 - 4 } & Min & Exp & Max \\
\hline \multicolumn{3}{|c|}{ Customer conditions } \\
\hline Initial Available CM Workforce & 2 & 5 & 7 \\
\hline Initial Average CM Skill Level & 0,4 & 0,7 & 1 \\
\hline Time to Adjust CM WF & 5 & 15 & 30 \\
\hline Time to Adjust CM Skill Level & 10 & 30 & 40 \\
\hline CM Materials Effectiveness & 1 & 1,3 & 1,5 \\
\hline CM Intangible Effectiveness & 1 & 1,3 & 1,5 \\
\hline \multicolumn{3}{|c|}{ Supplier conditions } \\
\hline Time to Adjust SD Productivity & 5 & 15 & 30 \\
\hline $\begin{array}{l}\text { SD SLA (Minimum Level of } \\
\text { Service) }\end{array}$ & 0,95 & 0,99 & 1 \\
\hline Minimum SD Skill Level & 0,5 & 0,8 & 1 \\
\hline
\end{tabular}

\subsection{Sensitivity of Impact Factors}

The sensitivity charts generated by the Vensim DSS simulation environment allow an intuitive visual analysis where it is possible to see the magnitude of the impacts caused by the realization of risk conditions at different confidence intervals. Without any thorough statistical analysis, one can observe the 
cumulative probability of an impact factor exceeding an expected value.

Figure 5 shows the variation of the impact factor Cumulative Cost of SD Capability. Figure 6 shows the variation of the impact factor Cumulative Cost of Insourced CM Capability.

In all 200 simulations performed for the sensitivity analysis, the Latin Hypercube sampling technique with a default noise seed (1234) and triangular probability distributions with the ranges in table 2 were used to generate the multivariate random sample of risk factors. The Latin Hypercube sampling ensures that the full range of each parameter being varied is explored more evenly and consistently in the simulations.

The solid line (peak) is the simulation result for the scenario in which all risk factors simultaneously assume their expected values. It is the baseline for the risk analysis. The dashed line in Figure 6 indicates the median of values for the Cumulative Cost of Insourced CM Capability.

The shaded areas represent the confidence intervals adopted for the sensitivity analysis, where $50 \%$ (light gray), $75 \%$ (gray), 95\% (dark gray) and $100 \%$ (black) of simulated impact factors are located. The limits of the black area represent the situation of the maximum and minimum impacts on service performance.

Each simulation runs for 400 days. This end-ofsimulation condition was adopted because this time period holds more than double the estimated simulated SO completion time and it is possible to observe all behaviors of interest.

Besides the sensitivity charts, the simulator generates histogram charts and the main statistical estimators (mean, median, standard deviation, minimum value, maximum value, normalized standard deviation). This information enables various statistical analyses on the impact factors, including the identification of their probability distribution functions.

Note that Figures 5 and 6 show, besides the dynamic behavior of the output variables over time, the variation of such behavior in response to variation (simultaneously or not) of the input parameters.

\subsection{Analysis of Simulation Results}

Following the risk management cycle, the information gathered from the sensitivity analysis (the risk assessment) can be used to prioritize risks, invest in risk prevention, risk control and risk mitigation activities. It is possible to calculate potential financial losses and to quantify indicators that can be used to support qualitative or subjective management decisions.

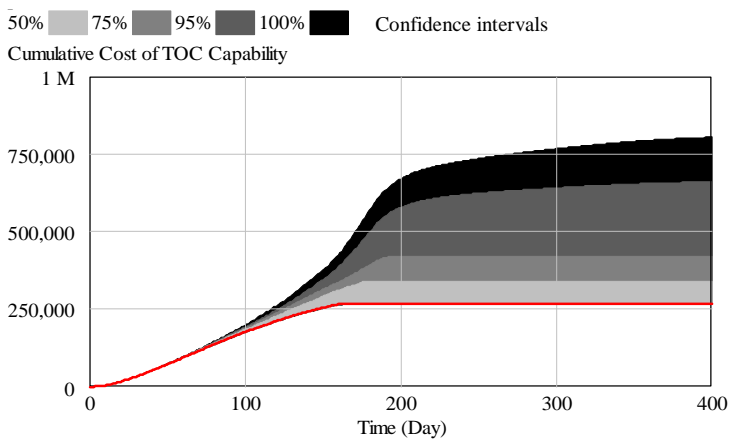

Figure 5: Cost of service $($ peak $=\$ 267,560)$.

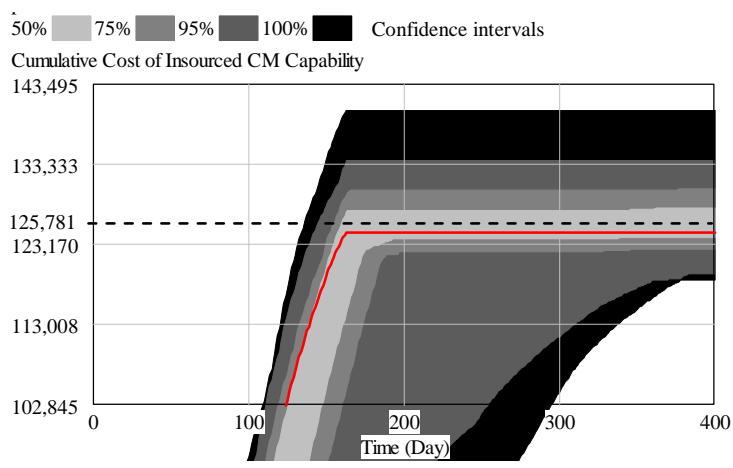

Figure 6: Total contract monitoring cost (peak $=\$ 124,609)$. The dashed line indicates the median.

To better understand the impacts of risk factors, we divided the sensitivity analysis into two subsections. First, we recorded the effects of uncertainty about the contracting organization's conditions over the impact factors. Then, we recorded the impacts caused by uncertainty about the supplier's conditions.

The impacts will arise when the expected values (peak line) for the impact factors are exceeded. The polarity of the relationship between risk factors and impact factors will define in which region of the graph undesirable values will be located. For instance, if $x$ is the expected cost of a service order to the supplier and $\mathrm{F}(x)$ the probability distribution function associated with this cost, the probability of service budget overrun is $1-\mathrm{F}$ ( $x \leq$ expected cost). $\mathrm{F}$ can be identified as the Chi-Square function from the frequency distribution of outputs using goodness-of-fit tests. 


\subsubsection{Varying the customer's conditions}

In total, 200 simulations were performed in which all model parameters related to the contract monitoring capability of the ITO customer organization varied simultaneously.

\subsubsection{Impact on service order cost}

Figure 5 shows the cost accumulation of the capability employed to execute the service order. Analyzing the behavior of the peak line, its shape shows a slight exponential growth in the initial phase of the simulation, during which the service delivery capability is being allocated and used. The inflection point indicates the time when this capability begins to be demobilized and its accumulated costs tend to stabilize (stop growing). This fact indicates that the service order has been fully executed. In terms of sensitivity analysis, the location of the peak line in relation to confidence intervals reveals a very unfavorable prognosis for the execution of the service order within the planned cost. Visually, one can estimate that between $50 \%$ and $75 \%$ of the simulation results exceed the budget for the service order. It is interesting to note in Figure 5 that the confidence intervals for $50 \%$ and $75 \%$, the output variable reaches a stable value within the simulated period (400 days). Not so in the confidence intervals of $95 \%$ and $100 \% 400$ days were not enough to conclude the SO. We increased the simulation period for up to 1000 days and the cost variable reached the stable state for these confidence intervals. Table 3 shows the confidence bounds for the cost of the SO.

Table 3: Confidence bounds for the cost of service.

\begin{tabular}{|c|r|r|}
\hline Conf. Bound & Min Cost & Max Cost \\
\hline $50 \%$ & $\$ 270,690$ & $\$ 341,580$ \\
\hline $75 \%$ & $\$ 266,470$ & $\$ 420,400$ \\
\hline $95 \%$ & $\$ 265,900$ & $\$ 663,824$ \\
\hline $100 \%$ & $\$ 265,852$ & $\$ 803,276$ \\
\hline All peak & \multicolumn{2}{|c|}{$\$ 267,560$} \\
\hline
\end{tabular}

Figure 7 shows the dynamic changes in the customer's contract monitoring (CM) capability level caused by the variation of the parameters that comprise it. Analyzing the behavior of the peak line, its shape shows that the level of CM capability grows to its maximum point at the 60th day of simulation. The concavity of the curve indicates that this growth is happening in a balance loop, controlled by the variable Desired Insourced CM Capability which limits the amount of capability to be allocated. However it is important to emphasize that this limit is not fixed throughout the simulation period. The maximum point of the curve represents the time when the maximum capability required to run the SO was reached. This point is not necessarily a horizontal asymptote, as there may be still available resources that can be allocated on demand and generate more capability. After a period at the maximum point, the curve starts to descend indicating capability deallocation until the conclusion of the SO.

Regarding the sensitivity analysis it is observed that most unfavorable values (below the expected level) for this capability were generated before the expected completion of the SO, i.e., 163 days. This fact was caused by the generation sequence of input values taken by Latin Hypercube (LH) sampling algorithm and will influence the results obtained by the sensitivity analysis of the completion time of the OS. More about this fact in the next section.

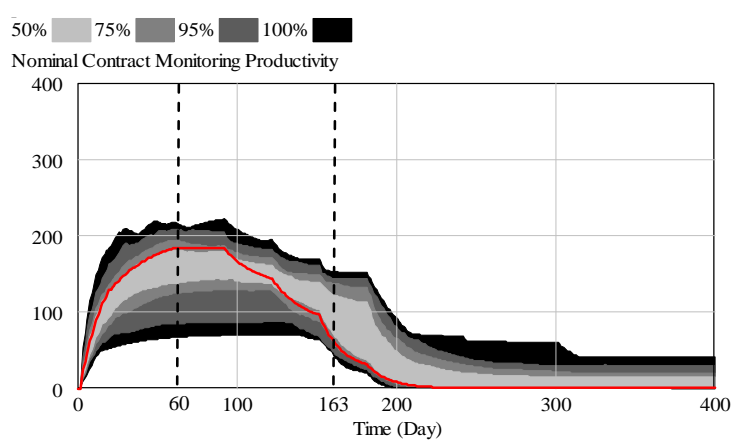

Figure 7: Dynamic changes in customer's contract monitoring capability.

Examining the histogram for the SO execution cost (not shown here) it was observed that the distribution resembles a negative exponential distribution. Therefore, given that the average of 200 simulations of the SO execution cost is $\$ 327,993$, the cumulative probability of a budget overrun is approximately $[1-\mathrm{P}(\mathrm{x} \leq \$ 267,560)]=55.77 \%$.

\subsubsection{Impact on service order conclusion time}

Table 4 shows that the expected conclusion time is reached only by the peak line. This behavior is not initially intuitive, producing the following question: why is the expected completion time never achieved even when risk factors assume favorable values? The answer lies in observing the locations between the confidence interval $50 \%$ to $75 \%$ related to the peak line in figure 7 . It is possible to realize higher contract monitoring capability values below the peak line near the 100th simulation day. 
Table 4: Confidence bounds for SO Conclusion Time.

\begin{tabular}{|c|c|c|}
\hline FIS07 & \multicolumn{2}{|c|}{ SO Conclusion Time } \\
\hline Conf. Bound & Min & Max \\
\hline $50 \%$ & 163 & 169 \\
\hline $75 \%$ & 163 & 177 \\
\hline $95 \%$ & 163 & 978 \\
\hline $100 \%$ & 163 & 980 \\
\hline All peak & \multicolumn{2}{|c|}{163} \\
\hline
\end{tabular}

This situation begins to reverse after the 100th day, when there is a higher concentration of favorable levels of this capability (above the peak line). However, this actually happens near the estimated SO completion time (day 163), causing a large concentration of simulation outcomes where the completion time of the OS is exceeded. This can be considered a "side effect" of the sequence of values that the Latin Hyperbole sampling algorithm generated for the variables that comprise the contract monitoring capability for some runs.

\subsubsection{Impact on contract monitoring cost}

For this intermediate risk factor, the relative position of the confidence intervals is more centralized, as shown in Figure 6, tending to a normal distribution. The peak line is slightly below the median $(\$ 125,781)$, indicating the predominance of unfavorable results in terms of cost. Assuming $\mathrm{F}(x)$ is a normal probability distribution, the cumulative probability of contract monitoring costs being higher than anticipated is $[1-\mathrm{F}(x \leq \$ 124,609)]=0.6543$, i.e., risk materializes in $65.43 \%$ of the simulations.

\subsubsection{Impact on rework}

Rework is a crucial stage for risk analysis in ITO and in general project management. Two model variables related to rework were analyzed: the number of penalties generated and the cost of rework. In $50 \%$ of the simulations the number of penalties converged to the expected value and in $75 \%$ the number deviated from the expected value by only one penalty. The same happened with the cost of rework. This implies that variations in contract monitoring capability will not impact the amount of generated rework but, rather, will influence the time taken to detect defects.

In a direct way, the factors related to rework impact the supplier financially and indirectly affect the relationship between customer and supplier. For all contracts analyzed within this model, the supplier is financially penalized because it bears the costs of penalties (not calculated here) and the operating cost of reworking.
We conclude this section by summarizing that the simulated uncertainty levels in the customer's conditions causes direct impacts on the service's conclusion time (IS2) and in contract management cost, which is an endogenous risk factor related to exceeding the budget for the service (IS1). Service conclusion time overruns can bring indirect impacts to the custmer, depending on how the outsourced service is related to the business layer. The high cost of contract management related tasks (IS3) is often overlooked by public organizations in Brazil since they do not include wages of the internal team responsible for this task in IT projects budgets.

Univariate simulations which vary the customer's risk factors one at a time were also undertaken in order to identify which had the most impact. The Initial Contract Monitoring Workforce factor is responsible for the greatest variation in the impact factors. Therefore, the model indicates that a more effective action to control or mitigate the risks of insufficient contract monitoring capability is to ensure sufficient human resources are allocated to this activity. Of course, the other components that comprise this capability are also important and should be considered when mitigating this risk. Investing in training and contract management tools, using methodologies and expertise to more accurately estimate the effort and cost of IT projects are actions that can mitigate risks associated with costs overrun.

\subsubsection{Varying the supplier's conditions}

200 simulations were performed varying simultaneously all model parameters related to the supplier's service delivery capability, namely: Time to Adjust SD productivity, Minimum SD Skill Level and SD SLA (Service Level Agreement).

Surprisingly, changes imposed on the supplier's conditions did not cause significant changes in the cost of the service, the cost of contract monitoring or in the service conclusion time. These factors exceeded expected values by up to $16 \%, 8 \%$ and $12 \%$ respectively.

The most significant impacts were on the number of penalties incurred and the cost of rework. These factors more than doubled in $70 \%$ of simulations and, in the worst case increased by up to $400 \%$ compared to the expected value. The risk factor to which impact factors were most sensitive was the Service Level Agreement, associated with the overall quality of service provided in relation to the percentage of defects generated. 
The most subjective impact being predicted (IS4: premature contract termination caused by low profitability for the supplier) has a high probability of arising in this scenario based on the indicators chosen here to represent the quality of the relationship between customer and supplier.

The simulations performed can provide multiple insights for decision makers regarding prevention and control of premature contract termination, which may compromise the quality of services and the achievement of planned benefits. The effects of a supplier's low service delivery capability go beyond the obvious and immediate delay in projects. They can compromise the quality of the relationship and the profitability of the contract, affecting the supplier itself, which could not withstand such impacts for long.

It is important that the customer monitors its suppliers' level of satisfaction in order to anticipate scenarios where switching supplier is needed typically a complex and costly process in Brazilian public organizations.

\subsection{Risk Management at SEFAZ and the proposed model}

Risk management at SEFAZ is currently carried out with a tool that uses a qualitative approach based on compliance. In this tool, a governance map is built associating objects in 3 different layers: business process in the strategic layer, IT processes in the tactical layer and IT asset (material resources, systems and human resources) in the operational layer. Qualitative levels of importance (low, medium, high) are assigned to each of the connections between objects. A set of controls is associated with each category of IT assets. These controls represent risk factors to which each asset is subject. The process of risk assessment with this tool consists in informing whether or not the controls are implemented. At the end of this process, qualitative risk indices (very low, low, medium, high, very high) are calculated for each asset and propagated to the strategic layer through the links defined on the governance map.

We have interviewed four users of such tool (an information security officer, an IT manager, a business process manager and a software project manager) on the tool's utility for them. They think the way risks are currently measured / reported suffices to prioritize them based on the indices. On the other hand, the qualitative nature of such indices does not allow decision makers to estimate actual extent of impacts and thus precludes trade off analyses of investments when addressing those risks. They believe the proposed model will facilitate these estimation and analyses.

\section{CONCLUSIONS AND OUTLOOK}

In this paper we showed how our model to support decision making in ITO and in IT capabilities can be used to analyze and prioritize risks. Following the risk assessment procedure in (Pfahl, 2005) applied to the context of a Brazilian state tax and finance agency (SEFAZ-AL), we analyzed the impact of two kinds of risks (lack of contract monitoring capability in contracting organizations and lack of service delivery capability in suppliers) on the ITO budget, on the deadline for completion of services and on the relationship between contractor and supplier.

Our experiments indicate that a lack of contract monitoring capability in ITO contracting organizations directly impacts on service conclusion time and influences the cost of contract management, which is an endogenous risk factor related to exceeding the service budget. It was also found that low levels of service delivery capability in the supplier most significantly impact the cost of rework and the number of penalties. These endogenous factors influence supplier profitability and may induce early termination of the contract.

The base model used in this study underwent a complete validation cycle (see Bezerra et al., 2014). However our implementation of risk assessment is a new feature that has gone through the initial stages of validation and verification (tests of structure, tests of behavior). In order to complete validation of its utility for supporting risk-based decision making for ITO, the model needs to undergo new tests of learning with the same interviewed group of users, as well as a more comprehensive comparative study between our approach and the currently risk assessment approach used at SEFAZ.

\section{Acknowledgements}

Authors thank the Science Without Borders Program from CAPES/ Ministry of Education of Brazil (www.capes.gov.br) for partially funding this work. Comments and suggestions from Prof. Dr. Dietmar Pfahl from the University of Tartu, Estonia are also gratefully acknowledged. 


\section{REFERENCES}

Abdel-Hamid, T. K., Madnick, S. E. (1991) Software Project Dynamics: An Integrated Approach, PrenticeHall.

Bahli, B., Rivard, S. (2005) 'Validating measures of information technology outsourcing risk factors', Omega, 332, 175-187.

Barney B. (1991) 'Firm resources and sustained competitive advantage', Journal of Management, vol.17, pp.99-120.

Bezerra T.R., Medeiros V. R. (2013) 'Managing customers' IT capabilities in IT outsourcing over time: A system dynamic approach', Proceedings of the $8 \mathrm{th}$ IFIP/IEEE International Workshop on Businessdriven IT Management - BDIM.

Bezerra T.R., Sampaio, A., Moura, J.A. (2014) 'A system dynamics model to support strategic decision making on IT Outsourcing: A case study at a state revenue agency in Brazil', Proceedings of the 9th IFIP/IEEE International Workshop on Business-driven IT Management - BDIM.

Carvalho, S. (2009) 'Um processo para gestão de contratos de aquisição de serviços de desenvolvimento de software na administração pública', master dissertation, UFPE, Recife, Brazil, Sep. 2009.

Costa, M. D., Braga, J.L., Abrantes, L.A., Ambrósio, B.G. (2013) 'Support to the Decision Making Process in Human Resources Management in Software Projects Based on Simulation Models', Revista Eletrônica de Sistemas de Informação, v. 12, n. 1, jan-mai 2013, artigo 51 doi:10.5329/RESI.2013.1201005.

Cunha, Mônica C. (2011) 'Aspectos e Fatores da Terceirização de Sistemas de Informação no Setor Púbico: Um Estudo em Instituições Públicas de Alagoas". Tese de Doutorado. Universidade Federal de Pernambuco'. In Portuguese.

Earl, M.J.(1996) 'The Risk of Outsourcing IT', Sloan Management Review/Spring 1996.

Garousi, V., Khosrovian, K. and Pfahl, D. (2009) 'A customizable pattern-based software process simulation model: design, calibration and application', Softw. Process: Improve. Pract., 14(3): 165-180.

Gui-sem, W., Xiang-yang, L. (2010) 'The Risk Analysis on IT Service Outsourcing of Enterprise with System Dynamics', International Conference on Service Sciences, DOI 10.1109/ICSS.2010.47.

Houston, D. X., Mackulak, G. T., Collofello, J. S. (2001) 'Stochastic simulation of risk factor potential effects for software development risk management', JSS 59(3), 247-257.

International Standards Organization (2008) Risk Management: Principles \& Guidelines on Implementation.

Jorfi, S., Nor, K. and Najjar, L. (2011) 'Assessing the impact of IT connectivity and IT capability on ITbusiness strategic alignment: an empirical study', Computer and Information Science, vol. 4, pp. 76-87.

Lacity, M. C., Khan, S. A., Willcocks, L. P. (2009) 'A review of the IT outsourcing literature: Insights for practice', The Journal of Strategic Information Systems, 18(3), 130-146. Elsevier B.V.

Lacity, M.,Khan, S., Yan, A., Willcocks, L. (2010) 'A review of the IT outsourcing empirical literature and future research directions', Journal of Information Technology, v.25, pp. 395-433.

Lin, C.Y., Abdel-Hamid, T.K., Sherif, J.S. (1997) 'Software-Engineering Process Simulation Model' (SEPS). JSS 38, 263-277.

Martens B., Teuteberg F. (2009) 'Why risk management matters in IT outsourcing: a literature review and elements of a research agenda', Proceedings of the 17th European Conf. on Information Systems, p. 1-13.

Ngwenyama, O. K., Technology, I., Sullivan, W. E., Patricia, B. (2006) 'Secrets of a Successful Outsourcing Contract: A Risk Analysis Framework for Analysing Risk Factors. Technology, (416), 1-12.

Ngwenyama, O. K., \& Sullivan, W. E. (2007) Outsourcing contracts as instruments of risk management: insights from two successful public contracts', Journal of Enterprise Information Management, 20(6), 615-640.

Osei-Bryson, K.M., Ngwenyama, O.K. (2006) 'Managing risks in information systems outsourcing: An approach to analyzing outsourcing risks and structuring incentive contracts', European Journal of Operational Research, 174 (1), 245-264.

Pfahl D. (2005) 'ProSim/RA - Software Process Simulation in Support of Risk Assessment'. In: Biffl S, Aurum A, Boehm B, Erdogmus H, Grünbacher P (eds.) Value-based Software Engineering, Springer Press, Berlin, pp 263-286.

Rivard, S., Albert, B., Patry, M. (1998) 'Assessing the Risk of IT Outsourcing', IEEE, 1998.Rivard, S., Albert, B., Patry, M., Dussault, S. (1998) 'Managing the Risk of IT Outsourcing', IEEE, Montreal.

Tan, B., Anderson Jr., E.G., Dyer, J.S. and Parker, G.G. (2010) 'Evaluating system dynamics models of risky projects using decision trees: alternative energy projects as an illustrative example', System Dynamics Review, vol 26, No 1, pp 1-17, January-March, 2010.

Willcocks, L. P., Lacity, M. C., Kern, T. (2000) 'Risk mitigation in IT outsourcing strategy revisited: longitudinal case research at LISA', Information Systems, 8(1999), 285-314. 